OPEN ACCESS

Edited by:

Xuguang (Sean) Li,

Health Canada, Canada

Reviewed by:

Roger Y. Tam,

Health Canada, Canada

Michel Gillbert,

National Research Council Canada (NRC-CNRC), Canada

*Correspondence:

Suh-Chin Wu

scwu@mx.nthu.edu.tw

${ }^{\dagger}$ These authors have contributed equally to this work

Specialty section:

This article was submitted to Vaccines and Molecular Therapeutics,

a section of the journal

Frontiers in Immunology

Received: 15 October 2021 Accepted: 17 November 2021 Published: 02 December 2021

Citation:

Lin W-S, Chen I-C, Chen H-C, Lee Y-C and Wu S-C (2021) Glycan Masking of Epitopes in the NTD and RBD of the Spike Protein Elicits Broadly Neutralizing Antibodies Against SARS-CoV-2 Variants.

Front. Immunol. 12:795741. doi: 10.3389/fimmu.2021.795741

\section{Glycan Masking of Epitopes in the NTD and RBD of the Spike Protein Elicits Broadly Neutralizing Antibodies Against SARS-CoV-2 Variants}

\author{
Wei-Shuo Lin ${ }^{1+}$, I-Chen Chen ${ }^{1+}$, Hui-Chen Chen ${ }^{1}$, Yi-Chien $L^{2} e^{2}$ and Suh-Chin Wu ${ }^{1,3^{*}}$ \\ 1 Institute of Biotechnology, National Tsing Hua University, Hsinchu, Taiwan, ${ }^{2}$ Department of Infectious Diseases, Fu Jen \\ Catholic University Hospital, New Taipei City, Taiwan, ${ }^{3}$ Department of Medical Science, National Tsing Hua University, \\ Hsinchu, Taiwan
}

Glycan-masking the vaccine antigen by mutating the undesired antigenic sites with an additional $N$-linked glycosylation motif can refocus B-cell responses to desired epitopes, without affecting the antigen's overall-folded structure. This study examined the impact of glycan-masking mutants of the N-terminal domain (NTD) and receptor-binding domain (RBD) of SARS-CoV-2, and found that the antigenic design of the $S$ protein increases the neutralizing antibody titers against the Wuhan-Hu-1 ancestral strain and the recently emerged SARS-CoV-2 variants Alpha (B.1.1.7), Beta (B.1.351), and Delta (B.1.617.2). Our results demonstrated that the use of glycan-masking Ad-S-R158N/Y160T in the NTD elicited a 2.8-fold, 6.5-fold, and 4.6-fold increase in the IC-50 NT titer against the Alpha (B.1.1.7), Beta (B.1.351) and Delta (B.1.617.2) variants, respectively. Glycan-masking of Ad-S-D428N in the RBD resulted in a 3.0-fold and 2.0-fold increase in the IC-50 neutralization titer against the Alpha (B.1.1.7) and Beta (B.1.351) variants, respectively. The use of glycan-masking in Ad-S-R158N/Y160T and Ad-S-D428N antigen design may help develop universal COVID-19 vaccines against current and future emerging SARS-CoV-2 variants.

Keywords: SARS-CoV-2, variant, glycan masking, COVID-19, vaccine

\section{INTRODUCTION}

Severe acute respiratory syndrome coronavirus 2 (SARS-CoV-2) is a coronavirus that can evolve within an infected host as a quasi-species with many mutations (within-host variants) arising for virus survival (1). Multiple SARS-CoV-2 variants have already emerged and are circulating globally, four of which are a cause of serious public health concern, also known as variants of concern: (i) Alpha (B.1.1.7 lineage) variant which originated in the United Kingdom (UK), (ii) Beta (B.1.351 lineage) variant which originated in South Africa, (iii) Gamma (P.1 lineage) variant which originated in Brazil, and (iv) Delta (B.1.617.2 lineage) variant which originated in India (2). The Alpha (B.1.1.7) variant encodes an S protein with nine mutations (del 69-70, Del 144, N501Y, 
A570D, D614G, P681H, T716I, S982A, and D1118H), of which $\mathrm{N} 501 \mathrm{Y}$ is in the receptor-binding domain (RBD). The Beta (B.1.351) variant encodes an $\mathrm{S}$ protein with nine mutations (L18F, D80A, D215G, Del 241-243, K417N, E484K, N501Y, D614G and A701V, three of which (K417N, E484K and $\mathrm{N} 501 \mathrm{Y}$ ) are in the RBD. The Gamma P.1 variant encodes an S protein with 12 mutations (L18F, T20N, P26S, D138Y, R190S, K417N/T, E484K, N501Y, D614G, H655Y, T1027I, and $\mathrm{V} 1176 \mathrm{~F})$, two of which are in the RBD (E484K, and N501Y). The Delta (B.1.617.2) variant encodes an $\mathrm{S}$ protein with ten mutations (T19R, G142D, del 156, del 157, R158G, L452R, T478K, D614G, P681R, and D950N), two of which are in the RBD (L452R and T478K). The immunity-evading mutations in the Beta (B1.351) variant include $\mathrm{E} 484 \mathrm{~K}$ in the RBD of the S protein (3), while those in the Delta (B.167.2) variant include L19R, del 157, del 158, L452R, and T478K in the S protein (4-6). In particular, the RBD mutations K417, L452, E484 and N501 may directly form a new interaction with the human angiotensin-converting enzyme 2 (ACE2) receptor $(7,8)$. Thus, the emerging SARS-CoV-2 B.1.351 variant can evade antibodymediated immunity without affecting the virus fitness and disease, as recently reported using convalescent plasma, vaccine-induced sera, and monoclonal antibodies (9-15). Whether the current authorized or approved vaccines can still provide effective protection against current and future emerging SARS-CoV-2 variants remains unclear.

Glycan-masking the vaccine antigen by mutating the undesired antigenic sites with an additional $N$-linked glycosylation motif can refocus the B-cell responses to the desired epitopes, without affecting the antigen's overall-folded structure $(16,17)$. This antigen design strategy has been used to develop vaccines against human immunodeficiency virus (HIV)1 (18-23), influenza virus (24-27), dengue and Zika viruses (28), and Middle East respiratory syndrome coronavirus (29). In this study, we used an adenovirus (Ad) vector encoding the fulllength $S$ gene of the SARS-CoV-2 Wuhan-Hu-1 isolate with a series of site-specific glycan-masking mutations on the $\mathrm{N}$ terminal domain (NTD) and RBD in a mouse immunization model, and then investigated the breadth of neutralizing antibodies elicited against SARS-CoV-2 and its Alpha (B.1.1.7), Beta (B.1.351), and Delta (B.1.617.2) variants. These results can provide useful information for the further development of universal coronavirus disease 2019 (COVID-19) vaccines against current and future emerging SARS-CoV-2 variants.

\section{MATERIALS AND METHODS}

\section{Cell Lines}

HEK293A and HEK293T cells were obtained from the Bioresource Collection and Research Center (BCRC), Taiwan. These cells were grown in Dulbecco's modified Eagle medium (DMEM) (Thermo Scientific) supplemented with 10\% fetal bovine serum (FBS) (Gibco) and 100 units/ml penicillin/ streptomycin $(\mathrm{P} / \mathrm{S})$, and maintained in an incubator at $37^{\circ} \mathrm{C}$ with $5 \% \mathrm{CO}_{2}$.

\section{Selection of Glycan-Masking Sites From the SARS-CoV-2 S Protein Structure}

Selection of the glycan-masking sites was based on the $3 \mathrm{D}$ structure of SARS-CoV-2 S protein (PDB ID:7C2L). The exposed loops or the protruding sites of the exposed loops on the NTD and $\mathrm{RBD}$ of the $\mathrm{S}$ protein were examined using PyMol (The PyMol Molecular Graphics System, version 4.0; Schrödinger, LLC). Glycan-masking sites that are less than $5 \AA$ from the native glycans and RBD were discarded.

\section{Preparation of Ad Vectors Expressing SARS-CoV-2 S Gene and Glycan- Masking Mutants}

The human codon-optimized S gene of SARS-CoV-2 (Wuhan$\mathrm{Hu}-1$ isolate, accession number MN908947.3) was obtained from GenScript. Site-directed mutagenesis was used to produce the glycan-masking $\mathrm{S}$ mutant genes, with the addition of an $\mathrm{N}$-linked glycosylation motif at the $\mathrm{S}$ protein residues $135 \mathrm{~N} / \mathrm{N} 137 \mathrm{~T}$, R158N/Y160T, N354/K356T, N370/A372T, G413N, D428N and H519N/P521T. Wild-type $S$ and glycan-masking $S$ genes were first cloned into the pENTR1A vector (Invitrogen), and then cloned into the adenoviral plasmid pAd/CMV/V5-DEST (Invitrogen) using LR ClonaseTM II Enzyme Mix (Invitrogen) to produce the Ad plasmid expressing SARS-CoV-2 S gene. To obtain Ad particles, the Ad plasmids were cleaved with Pac I restriction enzyme to expose the inverted terminal repeats and then transfected into 293A cells separately using TurboFect transfection reagent (Fermentas). After 10-15 d, once the cytopathic effects were visible, the transfected cells and culture media were collected. The cells were disrupted by means of three freeze-thaw cycles to release the intracellular viral particles, and the supernatants of the cell lysates were collected by centrifugation $\left(3000 \mathrm{rpm}, 15 \mathrm{~min}, 4^{\circ} \mathrm{C}\right.$ ) to obtain the $\mathrm{Ad}$ vectors expressing the SARS-Co-V-2 S proteins. To prepare higher titers, the virus was concentrated using a $30-\mathrm{kDa}$ Amicon Ultra-15 Centrifugal Filter (Millipore). The viral stocks were stored at $-80^{\circ} \mathrm{C}$. To determine the Ad titers, HEK293A cells were seeded into 6-well plates at a density of $10^{6}$ cells/well and incubated at $37^{\circ} \mathrm{C}$ overnight. The 10 -fold serially diluted Ad stocks were then added to each well at $37^{\circ} \mathrm{C}$ for $24 \mathrm{~h}$. Next, the media containing the diluted Ad vectors were removed, and $3 \mathrm{~mL} /$ well of DMEM containing $0.4 \%$ agarose and $100 \mathrm{U} / \mathrm{ml} \mathrm{P} / \mathrm{S}$ was added to the 6 -well plates. The plaques were visibly quantified 7-10 d after the cells were infected with Ad vectors, and the pfu count was noted.

\section{SDS-PAGE and Western Blot}

HEK293A cells were infected with Ad-S, Ad-S-F135N/N137T, Ad-S-R158N/Y160T, Ad-N354/K356T, Ad-S-N370/A372T, AdS-G413N, Ad-S-D428N and Ad-S-H519N/P521T at an MOI = 5 for $48 \mathrm{~h}$, then lysed with Glo Lysis buffer (Promega), and subjected to centrifugation at $12000 \times g$ for $5 \mathrm{~min}$ at $4^{\circ} \mathrm{C}$ to remove the cell debris. The lysates were heated at $95^{\circ} \mathrm{C}$ for $5 \mathrm{~min}$ in reducing sample buffer for $2 \mathrm{~h}$ at $37^{\circ} \mathrm{C}$, and resolved using $10 \%$ or $7 \%$ SDS-PAGE gels. For western blots, nitrocellulose membranes (Millipore) were blocked in 5\% (w/v) skim milk 
for $1 \mathrm{~h}$ at room temperature, followed by three washes with Trisbuffered saline containing $0.05 \%$ Tween 20 (TBST). SARS-CoV$2 \mathrm{~S}$ proteins were probed with anti-SARS-CoV-2 primary antibodies (GTX135356, GeneTex) overnight at $4^{\circ} \mathrm{C}$, and detected with horseradish peroxidase (HRP)-conjugated goat anti-rabbit IgG (KPL) for $1 \mathrm{~h}$ at room temperature. HRPcatalyzed enhanced chemiluminescence (Millipore) was captured using an X-ray film.

\section{Mouse Immunization}

Groups of female BALB/c mice (6 to 8 weeks old) ( $n=5$ per group) were obtained from the National Laboratory Animal Center, Taipei, Taiwan. Groups of female BALB/c mice (6 to 8 weeks old) ( $\mathrm{n}=5$ per group) were immunized with Ad-S, Ad-SF135N/N137T, Ad-S-R158N/Y160T, Ad-S-N370/A372T, or AdS-H519N/P521T vectors at $5 \times 10^{7}$ plaque-forming unit (pfu) per dose in PBS ( $\mathrm{pH}$ 7.4) in the first set of immunization experiments, and immunized with Ad-S, Ad-S-N354/K356T, Ad-S-G413N, and Ad-S-D428N vectors at $1 \times 10^{8}$ pfu per dose in the second set of immunization experiments. Intramuscular injections were administered at weeks 0 and 3. Sera were collected 2 weeks after the second immunization dose.

\section{Enzyme-Linked Immunosorbent Assay (ELISA)}

To measure the SARS-CoV-2 specific total IgG titer in the antisera, recombinant S (Wuhan-Hu-1, catalog number 40589V08H4), RBD (Wuhan-Hu-1, catalog number 40592-V08H), S1 (B.1.1.7 variant, catalog number 40591-VH12), RBD (B.1.1.7 variant, catalog number 40592-V08H82), S1 (B.1.351 variant, catalog number 40591-V08H10), RBD (B.1.351 variant, catalog number 40592-V08H85), S1 (B.1.617.2 variant, catalog number 40591-V49H2-B), RBD (B.1.617.2 variant, catalog number 40592-V08H90) proteins were obtained from Sino Biological Inc., and allowed to coat 96-well plates at a concentration of $2 \mu \mathrm{g} /$ $\mathrm{mL}$ in coating buffer $(100 \mu \mathrm{L} /$ well $)$ overnight at $4^{\circ} \mathrm{C}$. Coating buffers were aspirated and washed three times with PBS containing $0.05 \%$ Tween 20 (PBST). Each well was blocked with $200 \mu \mathrm{L}$ blocking buffer (1\% BSA in PBST) at room temperature for $2 \mathrm{~h}$. Heat-inactivated serum samples were prediluted $1: 1000$, followed by 2 -fold serial dilution in dilution buffer $(0.05 \%$ tween $20+1 \%$ BSA in PBST). The plates were washed three times with $300 \mu \mathrm{L}$ PBST (PBS with $0.05 \%$ Tween20 ), and then blocked with $200 \mu \mathrm{L}$ PBS buffer plus 1\% BSA for 2 $\mathrm{h}$ at room temperature, followed by three additional washes with $300 \mu \mathrm{L}$ PBST. Following this, the plates were incubated with 100 $\mu \mathrm{l}$ of HRP) conjugated anti-mouse IgG antibody (1:30000 in dilution buffer) for $1 \mathrm{~h}$ at room temperature. After three additional washes with $300 \mu \mathrm{L}$ PBST, $100 \mu \mathrm{L}$ of TMB substrate (BioLegend) was added to each well and incubated in the dark for $15 \mathrm{~min}$. The reaction was stopped by the addition of $100 \mu \mathrm{L}$ of 2 $\mathrm{N} \mathrm{H}_{2} \mathrm{SO}_{4}$. The optical density at $450 \mathrm{~nm}$ was measured using a TECAN spectrophotometer. The end-point titration values were calculated in terms of a final serial dilution higher than 0.2 optical density value.

\section{SARS-CoV-2 Pseudotyped Lentivirus Neutralization Assay}

To produce SARS-CoV-2 pseudoviruses, a plasmid expressing the full-length S protein (Wuhan-Hu-1, B.1.1.7, or B.1.351) of SARS-CoV-2 was co-transfected into HEK293T cells with packaging and reporter plasmids pCMV $\Delta 8.91$ and pLAS2w.FLuc.Ppuro (RNAi Core, Academia Sinica), using TransIT-LT1 transfection reagent (Mirus Bio). The medium was harvested and concentrated at $48 \mathrm{~h}$ post-transfection, followed by estimation of the pseudovirus titer in terms of the luciferase activity of SARS-CoV2-Spp transduction. Serum samples were serially diluted and incubated with 1,000 TU of SARS-CoV-2-pseudotyped lentivirus in DMEM (supplemented with $1 \% \mathrm{FBS}$ and $100 \mathrm{U} / \mathrm{mL} \mathrm{P} / \mathrm{S}$ ) for $1 \mathrm{~h}$ at $37^{\circ} \mathrm{C}$. The mixture was then inoculated with an equal volume of 10,000 HEK-293T cells stably expressing the ACE2 gene in 96-well plates, which were seeded one day before infection. The culture medium was replaced with fresh complete DMEM (supplemented with $10 \% \mathrm{FBS}, 100 \mathrm{U} / \mathrm{mL} \mathrm{P} / \mathrm{S}$ ) at $16 \mathrm{~h}$ post-infection and the cells were then continuously cultured for another $48 \mathrm{~h}$ before being subjected to a luciferase assay (Promega Bright-GloTM Luciferase Assay System). The percentage of inhibition was calculated as the ratio of the loss of luciferase readout (RLU) in the presence of serum to that of the no serum control. The formula used for the calculation was (RLU Control - RLU Serum)/RLU Control. Neutralization titers (IC-50) were measured as the reciprocal of the serum dilution required to obtain a $50 \%$ reduction in RLU compared to a control containing the SARS-CoV-2 S-pseudotyped lentivirus only. Neutralization curves and IC-50 values were analyzed using the GraphPad Prism 5 Software.

\section{Statistical Analyses}

Statistical tests for multiple comparison were performed for all groups (except for the PBS control) in case of the ELISA data. The results were analyzed using the nonparametric KruskalWallis test, with corrected Dunn's multiple comparison test, using GraphPad Prism v6.01. Statistical significance has been expressed as follows: ${ }^{*} \mathrm{p}<0.05$; ${ }^{* *} \mathrm{p}<0.01$; and ${ }^{* * *} \mathrm{p}<0.001$. Neutralization curves were fitted based on the equation of nonlinear regression log (inhibitor) vs. normalized response variable slope using GraphPad Prism v6.01. The IC-50 values of the neutralization were obtained from the fitting curves using GraphPad Prism v6.01

\section{RESULTS}

\section{Design of Engineered Glycan-Masking $S$ Antigens in the NTD and RBD for Ad Vector Immunization}

The $S$ protein of SARS-CoV-2 is trimeric, and each monomer comprises of S1 and S2 subunits (30-32). The S1 subunit contains NTD and RBD. To design glycan-masking $\mathrm{S}$ antigen(s) for immunization, we used an Ad vector encoding the full-length 
$\mathrm{S}$ gene of the SARS-CoV-2 Wuhan-Hu isolate, by introducing a series of $N$-linked glycosylation motifs into the $S 1$ region of the $S$ protein, to refocus the antibody responses to the $\mathrm{RBD}$ (Figure 1A). The sites of glycan-masking were introduced not only in the RBD, but also in the NTD, as RBD and NTD may spatially interact with each other in the quaternary structure of the intact trimeric $S$ protein (Figure 1B). The exposed loops or the protruding sites of the exposed loops on the NTD and RBD of the 3-D S protein structure (PDB ID: 7C2L) were chosen for the addition on the glycan-masking sites. Seven glycan-masking $\mathrm{N}$-glycan sites were engineered in the Ad-S vector: (\#1) Ad-SF135N/N137T, (\#2) Ad-S-R158N/Y160T, (\#3) Ad-S-N370/ A372T, (\#4) Ad-S-H519N/P521T, (\#5) Ad-S-N354/K356T, (\#6) Ad-S-G413N, and (\#7) Ad-S-D428N) (Figure 1B). To characterize the glycan- masking mutations on the $S$ protein, the lysates of HEK293A cells infected with each Ad-S vector were analyzed using $8 \%$ SDS-PAGE gels, followed by western blotting with an S1-specific polyclonal antibody. The results indicated the presence of S and S1 in the cell lysates of HEK293A cells infected with Ad-S, Ad-S-F135N/N137T, Ad-S-R158N/Y160T, Ad-SN354/K356T, Ad-S-N370/A372T, Ad-S-G413N, Ad-S-D428N, and Ad-S-H519N/P521T (Figure 2).

\section{Anti-S, Anti-RBD, and Pseudo-Neutralizing Antibody Titers Against the Wuhan-Hu-1 Ancestral Strain}

To investigate the antibody responses elicited by these Ad-S vectors, groups of $B A L B / c$ mice ( $n=5$ per group) were intramuscularly immunized with two doses of each Ad vector at $5 \times 10^{7}$ pfu per dose for the first set of immunization experiments and at $1 \times 10^{8}$ pfu per dose for the second set of immunization experiments all in a 3 -week interval; PBSimmunized animals were used as control. Sera from these immunized groups were collected after 2 weeks of the second dose and analyzed for the levels of anti-S, anti-RBD, and neutralizing antibodies against the original SARS-CoV-2 Wuhan-Hu-1 isolate. Two separate sets of immunization experiments were conducted in this study: (i) with Ad-S, Ad-SF135N/N137T, Ad-S-R158N/Y160T, Ad-S- N370/A372T, and Ad-S-H519N/P521T, and (ii) with Ad-S, Ad-S-N354/K356T, Ad-S-G413N, and Ad-S-D428N. In the first set of immunization experiments, the results indicated that the anti-S IgG titer elicited in the Ad-S- F135N/N137T-immunized group was significantly lower than those elicited in the wild-type Ad-S

A

N-linked glycans $\quad$ Glycan-masking
(original)

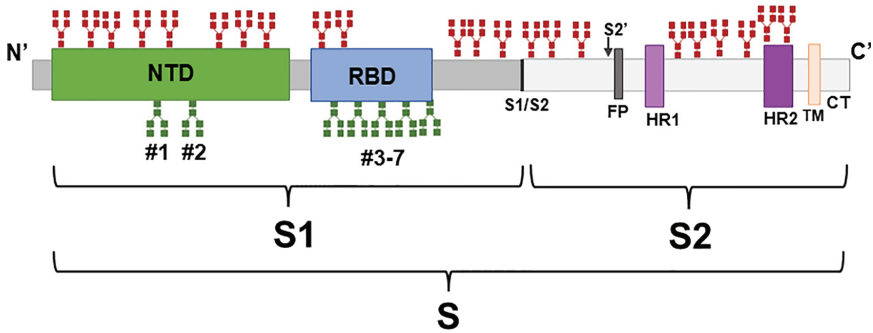

B

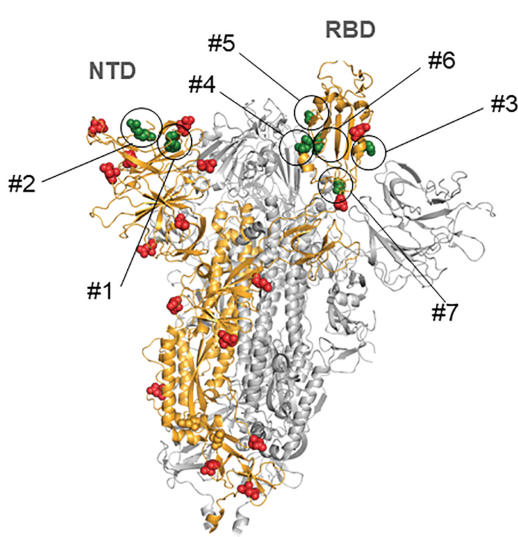

Glycan masking sites:

\#1 F135N/N137T

\#2 R158N/Y160T

\#3 N354/K356T

\#4 N370/A372T

\#5 G413N

\#6 D428N

\#7 H519N/P521T

FIGURE 1 | Schematic representation of SARS COV2 spike glycoprotein. (A) Positions of the original N-linked glycan (red) and the engineered N-linked glycan (green) amino-acid sequences shown as branches. Domains of the full-length S protein: $\mathrm{N}$-terminal domain (NTD), receptor binding domain (RBD), furin cleavage site (S1/S2), fusion peptide (FP), heptad repeat 1(HR1), heptad repeat 2 (HR2), transmembrane domain (TM), and cytoplasmic tail (CT); (B) The seven engineered Nlinked glycan sites of (\#1) F135N/N137T, (\#2) R158N/Y160T, (\#3) N354/K356T, (\#4) N370/A372T, (\#5) G413N, (\#6) D428N, and (\#7) H519N/P521T are shown in the intact trimeric $\mathrm{S}$ structure. 


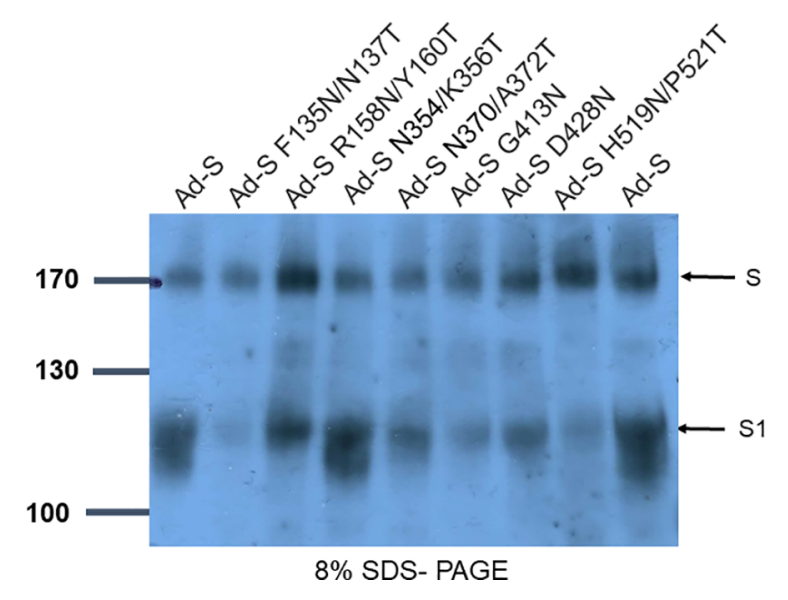

FIGURE 2 | Characterization of Ad-S glycan-masking mutants using SDSPAGE and western blotting. Cell lysates of HEK-293A cells infected with Ad-S, Ad-S-F135N/N137T, Ad-S-R158N/Y160T, Ad-S-N354/K356T, Ad-S-N370/ A372T, Ad-S-G413N, Ad-S-D428N, and Ad-S-H519N/P521T and analyzed using $8 \%$ SDS-PAGE and western blotting with an S1-specific polyclonal antibody. The results indicated the presence of $S$ and $S 1$ in the cell lysates.

and Ad-S-R158N/Y160T-immunized groups (Figure 3A). A reduced (but not statistically significant) titer of anti-RBD IgG antibodies was also observed for the Ad-S-F135N/N137Timmunized group, as compared to the other four immunized groups (Figure 3B). Pseudo-neutralizing antibody titers from the pooled sera of each group ( $n=5$ mice per group) were determined using the SARS-CoV-2 S (Wuhan-Hu-1)pseudotyped lentivirus assay in triplicate. In the first set of immunization experiments, Ad-S, Ad-S-F135N/N137T, Ad-SR158N/Y160T, Ad-S-N370/A372T, and Ad-S-H519N/P521Timmunized groups showed dose-response neutralization, while the PBS-immunized control group did not (Figure 3C). Antisera from the Ad-S-R158N/Y160T-immunized group showed increased neutralization potency, as compared to those of the Ad-S and Ad-S-N370/A372T-immunized groups, and the lower levels of the Ad-S-F135N/N137T and Ad-S-H519N/P521Timmunized groups (Figure 3C). The corresponding IC-50 titer elicited in the Ad-S-R158N/Y160T-immunized group against the Wuhan-Hu-1 ancestral strain was approximately 2.4-fold higher than that elicited in the wild-type Ad-S-immunized group (Figure 3D). No significant differences were observed in the anti-S and anti-RBD titers in the second set of immunization experiments with the Ad-S, Ad-S-N354/K356T, Ad-S-G413N, and Ad-S-D428N-immunized groups (Figures 3E, F). Dosedependent pseudo-neutralization curves were observed for the Ad-S, Ad-S-N370/K356T, Ad-S-G413N, and Ad-S-D428Nimmunized groups, but not for the PBS-immunized control (Figure 3G). The IC-50 titers of the Ad-S-N354/K356T and Ad-S-D428N-immunized groups against the Wuhan-Hu-1 ancestral strain were approximately 2.5 - and 2.8 -fold higher, as compared to that of the wild-type Ad-S-immunized group (Figure 3H). These results indicated that the glycan-masking Ad-S-R158N/Y160T in NTD and glycan-masking Ad-S-N354/
K356T and Ad-S-D428N in RBD elicited a 2.4-, 2.5-, and 2.8-fold increase, respectively, in the pseudo-neutralization IC-50 titer against the Wuhan-Hu-1 ancestral strain.

\section{Anti-S1, Anti-RBD, and Pseudo- Neutralizing Antibody Titers Against SARS-CoV-2 Alpha (B.1.1.7), Beta (B.1.351), and Delta (B.1.617.2) Variants}

To further study the neutralization against SARS-CoV-2 variants, the titers of anti-S1, and anti-RBD IgG, and neutralizing antibodies against the Alpha (B.1.1.7), Beta (B.1.351), and Delta (B.1.617.2) variants were measured using the same antisera. In the first set of immunization experiments in the Ad-S, Ad-S-F135N/N137T, Ad-S-R158N/Y160T, Ad-SN370/A372T, and Ad-S-H519N/P521T-immunized groups, we found that the anti-S1 IgG titers against Alpha (B.1.1.7) variant in the Ad-S-F135N/N137T-immunized group were lower than the wild type Ad-S and Ad-S- N370/A372T-immunized groups (Figure 4A). A reduced (but not statistically significant) titer of anti-RBD IgG antibodies against Alpha (B.1.1.7) variant was observed for the Ad-S-F135N/N137T-immunized group, as compared to the other four immunized groups (Figure 4B). The pseudovirus neutralization curve of the Ad-S-R158N/ Y160T-immunized group against the Alpha (B.1.1.7) variant was more potent than those of the wild-type Ad-S-immunized group and the three other immunization groups (Figure 4C), with an approximately 2.8 -fold increase in the neutralization IC50 titer, as compared to that of the wild-type Ad-S-immunized group (Figure 4D). In the second set of immunization experiments in the Ad-S, Ad-S-N354/K356T, Ad-S-G413N, and Ad-S-D428N-immunized groups, no significant differences were observed in the anti-S1 and anti-RBD titers among these four Ad immunization groups (Figures 4E, F). However, the AdS-D 428N-immunized group displayed more potent neutralization against the Alpha (B.1.1.7) variant than the three other groups (Figure 4G), resulting in a 3.0-fold increase in the neutralization IC-50 titer, as compared to that of the wildtype Ad-S-immunized group (Figure $\mathbf{4 H}$ ). Therefore, the glycanmasking Ad-S-R158N/Y160T in NTD and glycan-masking Ad$\mathrm{S}-\mathrm{D} 428 \mathrm{~N}$ in $\mathrm{RBD}$ were found to elicit increased titers of neutralizing antibodies against the Alpha (B.1.1.7) variant.

In the case of the Beta (B.1.351) variant in the first set of immunization experiments in the Ad-S, Ad-S-F135N/N137T, Ad-S-R158N/Y160T, Ad-S- N370/A372T, and Ad-S-H519N/ P521T-immunizd groups, the Ad-S-F135N/N137T-immunized group had a significantly lower IgG titer of anti-S1 antibodies than those in the wild type Ad-S and Ad-S-N370/A372Timmunized groups (Figure 5A). The anti-RBD IgG titer in the Ad-S-F135N/N137T-immunized group was lower, as compared to the titers in the Ad-S-R158N/Y160T and Ad-S-H519N/ P521T-immunizd groups (Figure 5B). Both the Ad-S-R158N/ Y160T and Ad-S-N370/A372T groups showed increased potency for pseudo-neutralization against the Beta (B.1.351) variant, as compared to the three other groups (Ad-S, Ad-S-F135N/N137T, and Ad-S-H519N/P521T-immunized) (Figure 5C); the neutralization IC-50 titer was approximately 6.5-fold and 


\section{Wuhan-Hu-1 (ancestral)}

A

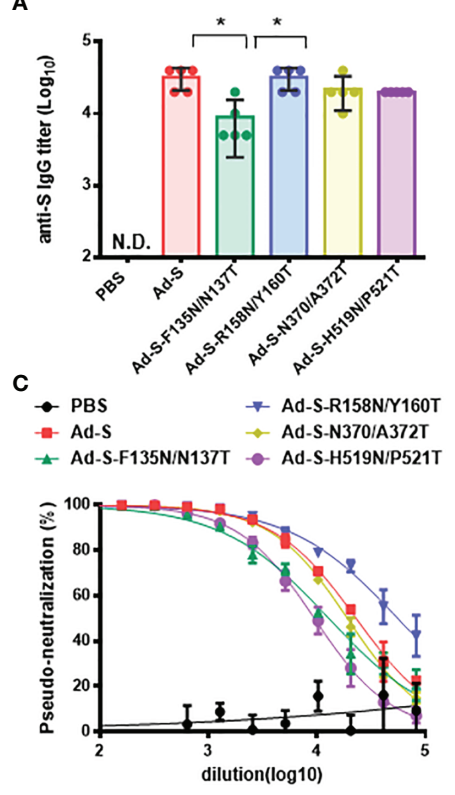

E

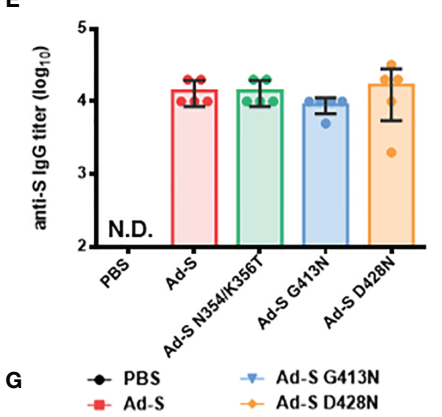

\-Ad-S N354/K356T

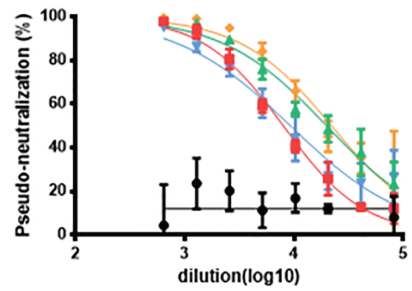

B

D
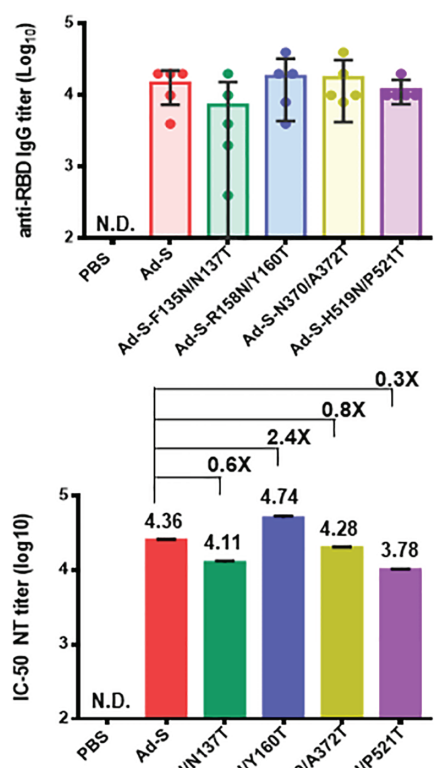

$\mathbf{F}$

H

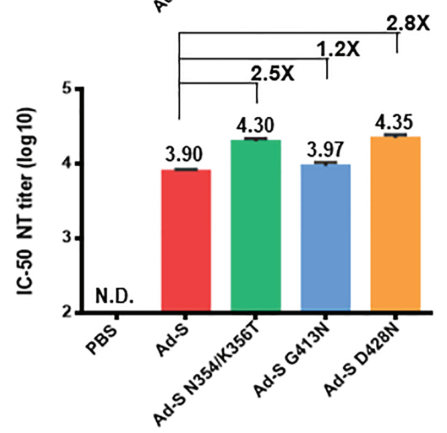

FIGURE 3 | Antibody responses elicited by Ad-S glycan-masking mutants against the SARS-CoV-2 Wuhan-Hu-1 strain. Ad-S immunization regimens in the first and second sets of immunization experiments: (A) antisera for anti-S IgG titers from each group of mice $(n=5)$ and tested individually in the first set of immunization experiments; (B) antisera for anti-RBD IgG titers from each group of mice $(n=5)$ and tested individually in the first set of immunization experiments; (C) the doseresponse pseudo-neutralization curves for the pooled sera of each immunized group of mice $(n=5)$ and measured in triplicate the first set of immunization experiments; (D) IC-50 NT titer for neutralizing antibodies against the Wuhan-Hu-1 ancestral strain in the first set of immunization experiments; (E) antisera for anti-S IgG titers from each group of mice $(n=5)$ and tested individually in the second set of immunization experiments; (F) antisera for anti-RBD IgG titers from each group of mice $(n=5)$ and tested individually in the second set of immunization experiments; $(\mathbf{G})$ the dose-response pseudo-neutralization curves for the pooled sera of each immunized group of mice $(n=5)$ and measured in triplicate the second set of immunization experiments; $(\mathbf{H})$ IC- 50 NT titer for neutralizing antibodies against the Wuhan-Hu-1 ancestral strain in the second set of immunization experiments. Fold-changes of the Ad-S glycan-masking mutant IC-50 NT titers against the wild-type Ad-S titer on a linear scale indicated. Statistical tests for multiple comparison of anti-S and anti-RBD IgG titers among the Ad-S immunization groups were performed using the nonparametric test with Kruskal-Wallis with corrected Dunn's multiple comparison. The statistical significance is expressed as follows: ${ }^{\star} p<0.05$. Neutralization curves were fitted based on the equation of nonlinear regression log (inhibitor) vs. normalized response - variable slope using GraphPad Prism v6.01. The IC-50 values of neutralization were obtained from the fitting curves using GraphPad Prism v6.01. Error bars are plotted as standard deviation from the mean value. Not detectable for N.D. 


\section{Alpha (B.1.1.7)}

A

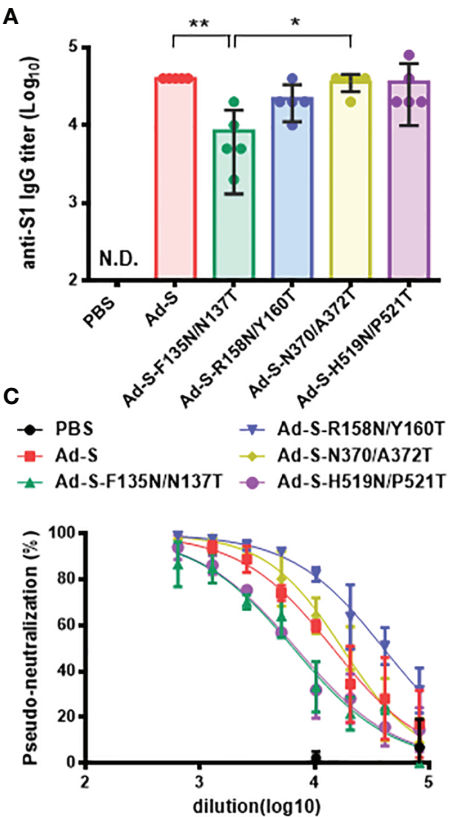

E
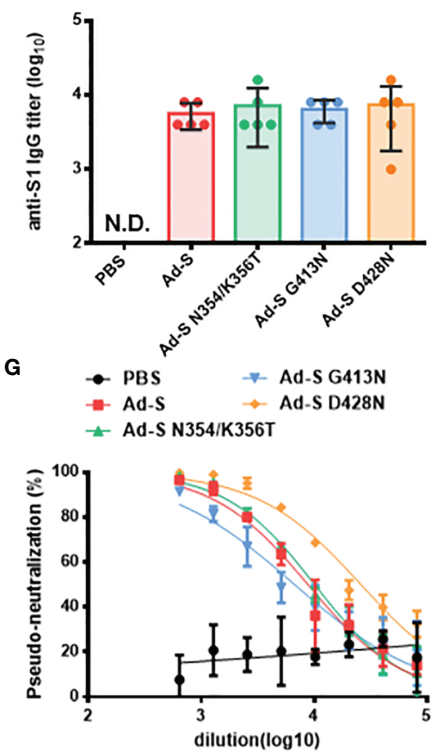
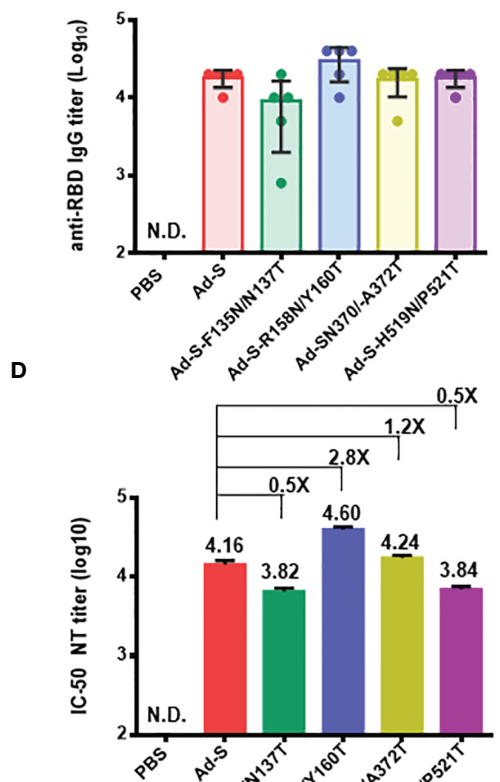

$\mathbf{F}$

$\mathbf{H}$

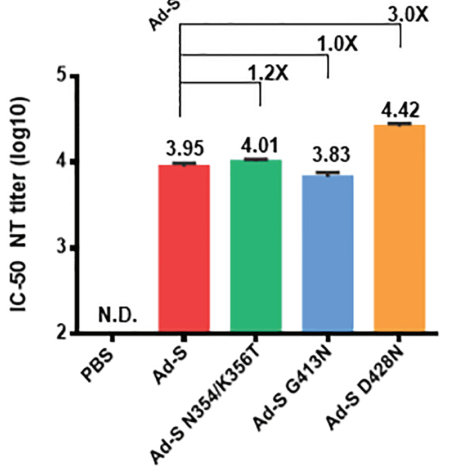

FIGURE 4 | Antibody responses elicited by Ad-S glycan-masking mutants against SARS-CoV-2 Alpha (B.1.1.7) variant. (A) antisera for anti-S1 IgG titers from each group of mice $(n=5)$ and tested individually in the first set of immunization experiments; (B) antisera for anti-RBD IgG titers from each group of mice ( $n=5)$ and tested individually in the first set of immunization experiments; (C) the dose-response pseudo-neutralization curves for the pooled sera of each immunized group of mice $(n=5)$ and measured in triplicate in the first set of immunization experiments; (D) IC-50 NT titer for neutralizing antibodies against Alpha (B.1.1.7) variant; (E) antisera for anti-S1 lgG titers from each group of mice $(n=5)$ and tested individually in the second set of immunization experiments; (F) antisera for anti-RBD lgG titers from each group of mice $(n=5)$ and tested individually in the second set of immunization experiments; (G) the dose-response pseudo-neutralization curves for the pooled sera of each immunized group of mice $(n=5)$ and measured in triplicate for the second set of immunization experiments; (H) IC-50 NT titer for neutralizing antibodies against Alpha (B.1.1.7) variant for the second set of immunization experiments. Fold-changes of the Ad-S glycan-masking mutant IC-50 NT titers against the wildtype Ad-S (Alpha, B.1.1.7 variant) titer on a linear scale are indicated. Statistical tests for multiple comparison of anti-S1 and anti-RBD titers among Ad-S immunization groups were performed using the nonparametric test with Kruskal-Wallis with corrected Dunn's multiple comparison. The statistical significance is expressed as follows: ${ }^{*} p<0.05$ and ${ }^{* *} p<0.01$. Neutralization curves were fitted based on the equation of nonlinear regression log (inhibitor) vs. normalized response - variable slope using GraphPad Prism v6.01. The IC-50 values of neutralization were obtained from the fitting curves using GraphPad Prism v6.01. Not detectable for N.D. Error bars are plotted as standard deviation from the mean value. 


\section{Beta (B.1.351)}

A

C

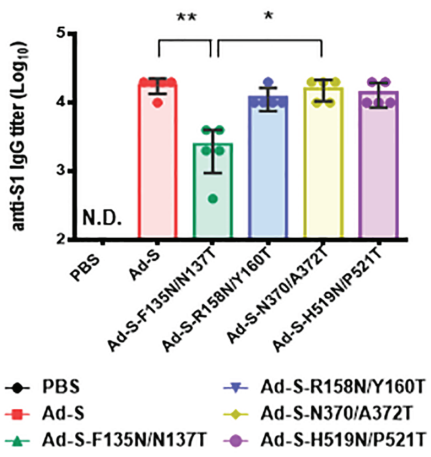

E

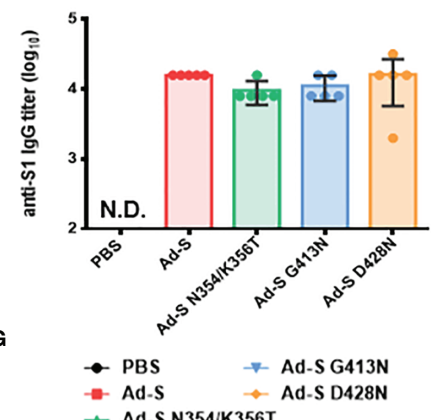

¿- Ad-S N354/K356T

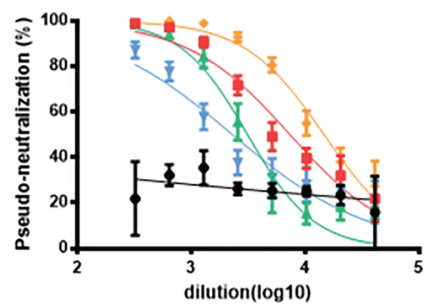

B

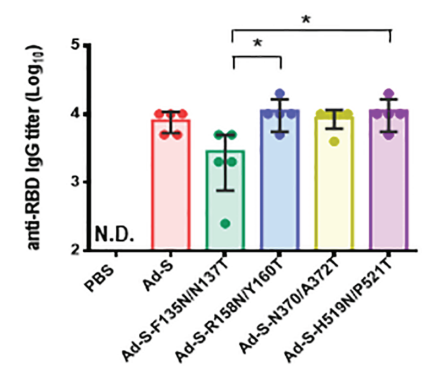

D
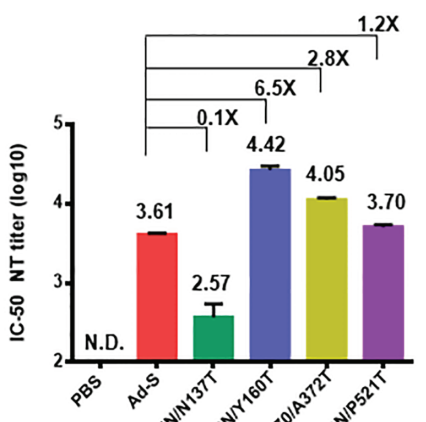

H
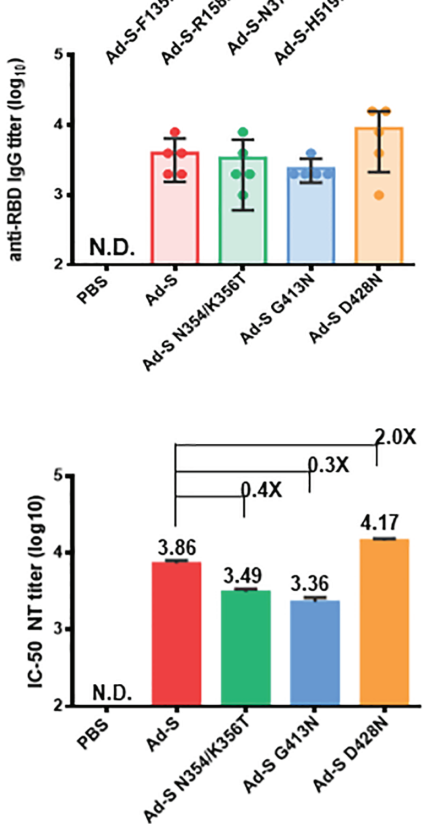

FIGURE 5 | Antibody responses elicited by Ad-S glycan-masking mutants against SARS-CoV-2 Beta (B.1.351) variant. (A) antisera for anti-S1 IgG titers from each group of mice $(n=5)$ and tested individually in the first set of immunizations; $(\mathbf{B})$ antisera for anti-RBD IgG titers from each group of mice ( $n=5)$ and tested individually in the first set of immunization experiments; (C) the dose-response pseudo-neutralization curves for the pooled sera of each immunized group of mice ( $\mathrm{n}=5$ ) and measured in triplicate for the first set of immunization experiments; (D) IC-50 NT titer for neutralizing antibodies against Beta (B.1.351 variant) for the first set of immunization experiments; (E) antisera for anti-S1 lgG titers from each group of mice $(n=5)$ and tested individually in the second set of immunizations; (F) antisera for anti-RBD IgG titers from each group of mice $(n=5)$ and tested individually in the second set of immunization experiments; (G) the dose-response pseudoneutralization curves for the pooled sera of each immunized group of mice $(n=5)$ and measured in triplicate for the second set of immunization experiments; $(\mathbf{H}) \mathbf{I C}$ 50 NT titer for neutralizing antibodies against Beta (B.1.351 variant) for the first set of immunization experiments. Fold-changes of the Ad-S glycan-masking mutant IC-50 NT titer against the wild-type Ad-S (Beta, B.1.351 variant) titer are indicated. Statistical tests for multiple comparison of anti-S1 and anti-RBD IgG titers among Ad-s immunization groups were performed using the nonparametric test with Kruskal-Wallis with corrected Dunn's multiple comparison. The statistical significance is expressed as follows: ${ }^{*} p<0.05$ and ${ }^{* \star} p<0.01$. Neutralization curves were fitted based on the equation of nonlinear regression log (inhibitor) vs. normalized response - variable slope using GraphPad Prism v6.01. The IC-50 values of neutralization were obtained from the fitting curves using GraphPad Prism v6.01. Error bars are plotted as standard deviation from the mean value. Not detectable for N.D. 
2.8-fold higher in the glycan-masking Ad-S-R158N/Y160T and Ad-S-N370/A372T-immunized groups, respectively (Figure 5D). In the second set of immunization experiments in the Ad-S, AdS-N354/K356T, Ad-S-G413N, and Ad-S-D428N-immunized groups, no significant differences were observed in the anti-S1 and anti-RBD titers among these four Ad immunization groups (Figures 5E, F). The Ad-S-D428N-immunized group was more potent in neutralizing the Beta (B.1.351) variant than the other three groups (Figure 5G), resulting in a 2.0-fold increase in the neutralization IC-50 titer, as compared to that of the wild-type Ad-S-immunized group (Figure 5H). Therefore, immunization with the glycan-masking Ad-S-R158N/Y160T in NTD and glycan-masking Ad-S-N370/A372T and Ad-S-D428N in RBD were more potent than that with the wild-type Ad-S in eliciting neutralizing antibodies against the Beta (B.1.351) variant.

In the case of Delta (B.1.617.2) variant, we found that the anti-S1 IgG titers in the Ad-S-F135N/N137T-immunized group were lower than the wild type Ad-S and Ad-S-R158N/Y160T,immunized groups (Figure 6A). No significant differences in anti-RBD IgG titers were observed among these Ad-immunized groups in the first set of experiments (Figure 6B). Both the Ad-SF135N/N137T and Ad-S-R158N/Y160T-immunized groups elicited more potent pseudo-neutralization against the Delta (B.1.167.2) variant, as compared to the Ad-S, Ad-S-N370/ A372T, and Ad-S-H519N/P521T-immunized groups, in the first set of immunization experiments (Figure 6C); an approximately 3.7 -fold and 4.6 -fold increase in the neutralization IC-50 titer was found for the glycan-masking Ad-S-F135N/N137T and Ad-S-R158N/Y160T groups, respectively (Figure 6D). In the second set of immunization experiments among the Ad-S, Ad-S-N354/K356T, Ad-S-G413N, and Ad-S-D428N-immunized groups, the pseudo-neutralization curves of the three glycan-masking groups against the Delta (B.167.2) variant were less potent than that of the wild-type AdS-immunized group (Figure 6G) with a reduced IC-50 titers to $0.46,0.23$, and 0.46 fold, respectively (Figure $6 \mathbf{H}$ ). Therefore, only the glycan-masking Ad-S-F135N/N137T and Ad-S-R158N/ Y160T in NTD elicited more potent neutralizing antibody titers against the Delta (B.1.617.2) variant.

\section{Comparison of Neutralization IC-50 Titers Elicited by Glycan-Masking Ad-S Mutants Against the Wuhan-Hu-1 Ancestral Strain}

To compare these results, the neutralizing IC-50 titers from the two separate sets of immunization experiments were normalized to the titer elicited by the wild-type Ad-S against the Wuhan-Hu1 ancestral strain from. In the first set of immunization experiments, the neutralization IC-50 titers elicited in the glycan-masking Ad-S-R158N/Y160T-immunized group showed a 2.5-fold increase against the Wuhan-Hu-1 ancestral strain, a 1.8-fold increase against the Alpha (B.1.1.7) variant, a 1.2-fold increase against the Beta (B.1.351) variant, but a 0.6-fold decrease against the Delta (B.1.617.2) variant (Figure 7A). The titer for the Ad-S-R158N/Y160T group against the Delta (B.1.617.2) variant was still higher than the titer of Ad-S against the Delta variant (Figure 7A). In the second set of immunization experiments, the neutralization IC-50 titers elicited in the Ad-S-D428N-immunized group showed a 2.7fold increase against the Wuhan-Hu-1 ancestral strain, a 3.2-fold increase against the Alpha (B.1.1.7) variant, a 2.0-fold increase against the Beta (B.1.351) variant, but a 0.2-fold decrease against the Delta (B.1.617.2) variant (Figure 7B). Therefore, the glycanmasking Ad-S-R158N/Y160T in NTD elicited more potent neutralizing antibodies against the Wuhan-Hu-1 ancestral strain and increased the cross-neutralizing antibody titers against the Alpha (B.1.1.7), Beta (B.1.351), and Delta (B.1.617.2) variants. The glycan-masking Ad-S-D428N in RBD also elicited more potent neutralizing antibodies against the Wuhan-Hu-1 ancestral strain but only increased the crossneutralizing antibody titers against the Alpha (B.1.1.7) and Beta (B.1.351) variants.

\section{DISCUSSION}

This study reported the use of glycan-masking antigen design by selective incorporation of an $\mathrm{N}$-linked glycosylation motif on the NTD and RBD in the SARS-CoV-2 S gene encoded by the Ad vector, to develop universal COVID-19 vaccines. It has been recently confirmed that the S protein of SARS-CoV-2 is heavily glycosylated, with approximately 22 potential $N$-glycan sites on each $\mathrm{S}$ monomer $(30,32,33)$. We introduced seven separate $\mathrm{N}$ linked glycosylation sites into the $\mathrm{S}$ glycoprotein, S-F135N/ N137T, S-R158N/Y160T, Ad-S- N354/K356T, S-N370/A372T, Ad-S-G413N, Ad-S-D428N and S-H519N/P521T, in the NTD and RBD. However, we were unable to demonstrate the addition of a single $\mathrm{N}$-glycosylation site for these glycan-masking Ad-S mutants using SDS-PAGE gel in western blots. It is possible that SDS-PAGE in the conditions used in this work does not have the resolution to show the addition of a single $N$-glycosylation site when there are already 22 sites. A more advanced use of the selected mutants would warrant thorough site-specific characterization by mass spectrometry. It is also possible that glycan-masking mutations may also affect the $S$ protein stabilization for cell surface expression, S/S1 cleavage, and surface $S$ expression. Thus, there is a need for further characterization of these glycan-masking mutants, particularly Ad-S-R158N/Y160T and Ad-S-D428N expressed S proteins.

Our results showed that the glycan-masking Ad-S-R158N/ Y160T at the N3 loop in the NTD and the glycan-masking Ad-S$\mathrm{N} 354 / \mathrm{K} 356 \mathrm{~T}$ at the C-3 loop in the RBD (Supplementary Figure 1) elicited a potent neutralizing antibody response against the WuhanHu-1 ancestral strain (Figure 3). Selection of these glycan-masking sites in this investigation was based on visual inspection of the 3-D S protein structure (PDB ID: 7C2L) for the exposed loops or the protruding sites of the exposed loops in NTD and RBD of the S1 subunit (Supplementary Figure 1). The increased IC-50 NT titers against the Wuhan-Hu-1 ancestral strain by the glycan-masking Ad-S-R158N/Y160T-immunized group correlated with the increased neutralization titers against the Alpha (B.1.1.7), Beta (B.1.351) and Delta (B.1.617.2) variant (Figures 4-6). Only the glycan-masking Ad-S-F135N/N137T and Ad-S-R158N/Y160T in 


\section{Delta (B.1.617.2)}

A

C

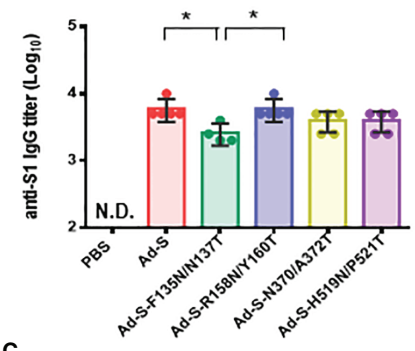

$\mathrm{C}$
$\stackrel{-}{-}$ PBS
- Ad-S

$\rightarrow$ Ad-S-R158N/Y160T

Ad-S-N370/A372T

$\star$ Ad-S-F135N/N137T $\bullet$ Ad-S.H519N/P521T

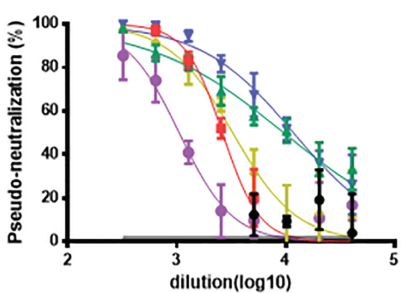

E

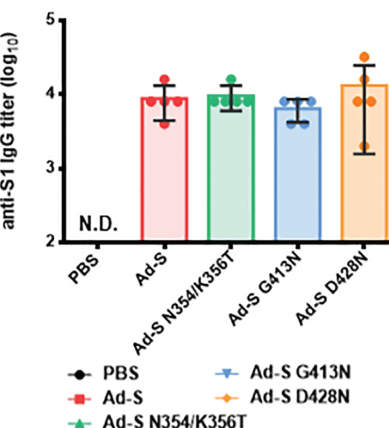

Ad-S N354/K356T

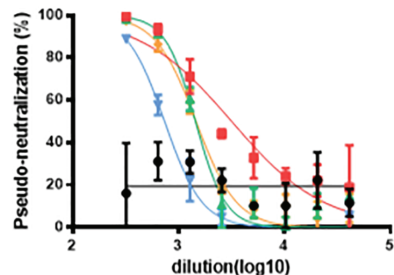

B

D
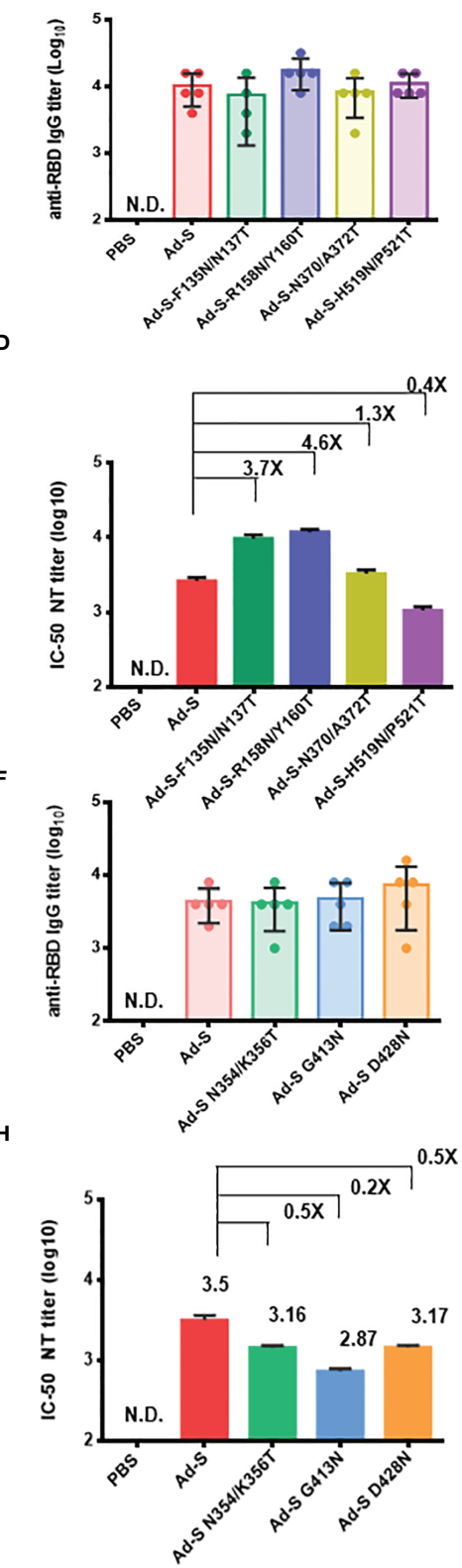

FIGURE 6 | Antibody responses elicited by Ad-S glycan-masking mutants against SARS-CoV-2 Delta (B.1.617.2) variant. (A) antisera for anti-S1 IgG titers from each group of mice $(n=5)$ and tested individually in the first set of immunization experiments; $(\mathbf{B})$ antisera for anti-RBD IgG titers from each group of mice ( $\mathrm{n}=5$ ) and tested individually in the first set of immunization experiments; (C) the dose-response pseudo-neutralization curves for the pooled sera of each immunized group of mice $(n=5)$ and measured in triplicate for the first set of immunization experiments; (D) IC-50 NT titer for neutralizing antibodies against Delta (B.1.617.2 variant) in the first set of immunization experiments; (E) antisera for anti-S1 lgG titers from each group of mice ( $\mathrm{n}=5$ ) and tested individually in the second set of immunization experiments; (F) antisera for anti-RBD lgG titers from each group of mice ( $n=5)$ and tested individually in the second set of immunization experiments; (G) the doseresponse pseudo-neutralization curves for the pooled sera of each immunized group of mice $(n=5)$ and measured in triplicate for the second set of immunization experiments; (H) IC-50 NT titer for neutralizing antibodies against Delta (B.1.617.2 variant) in the second set of immunization experiments. Fold-changes of the Ad-S glycan-masking mutant IC-50 NT titer against the wild-type Ad-S (Delta, B.1.617.2 variant) titer are indicated. Statistical tests for multiple comparison of anti-S1 and anti-RBD IgG titers among Ad-S immunization groups were performed using the nonparametric test with Kruskal-Wallis with corrected Dunn's multiple comparison. The statistical significance is expressed as follows: ${ }^{*} p<0.05$. Neutralization curves were fitted based on the equation of nonlinear regression log (inhibitor) vs. normalized response - variable slope using GraphPad Prism v6.01. The IC-50 values of neutralization were obtained from the fitting curves using GraphPad Prism v6.01. Error bars are plotted as standard deviation from the mean value. Not detectable for N.D. 


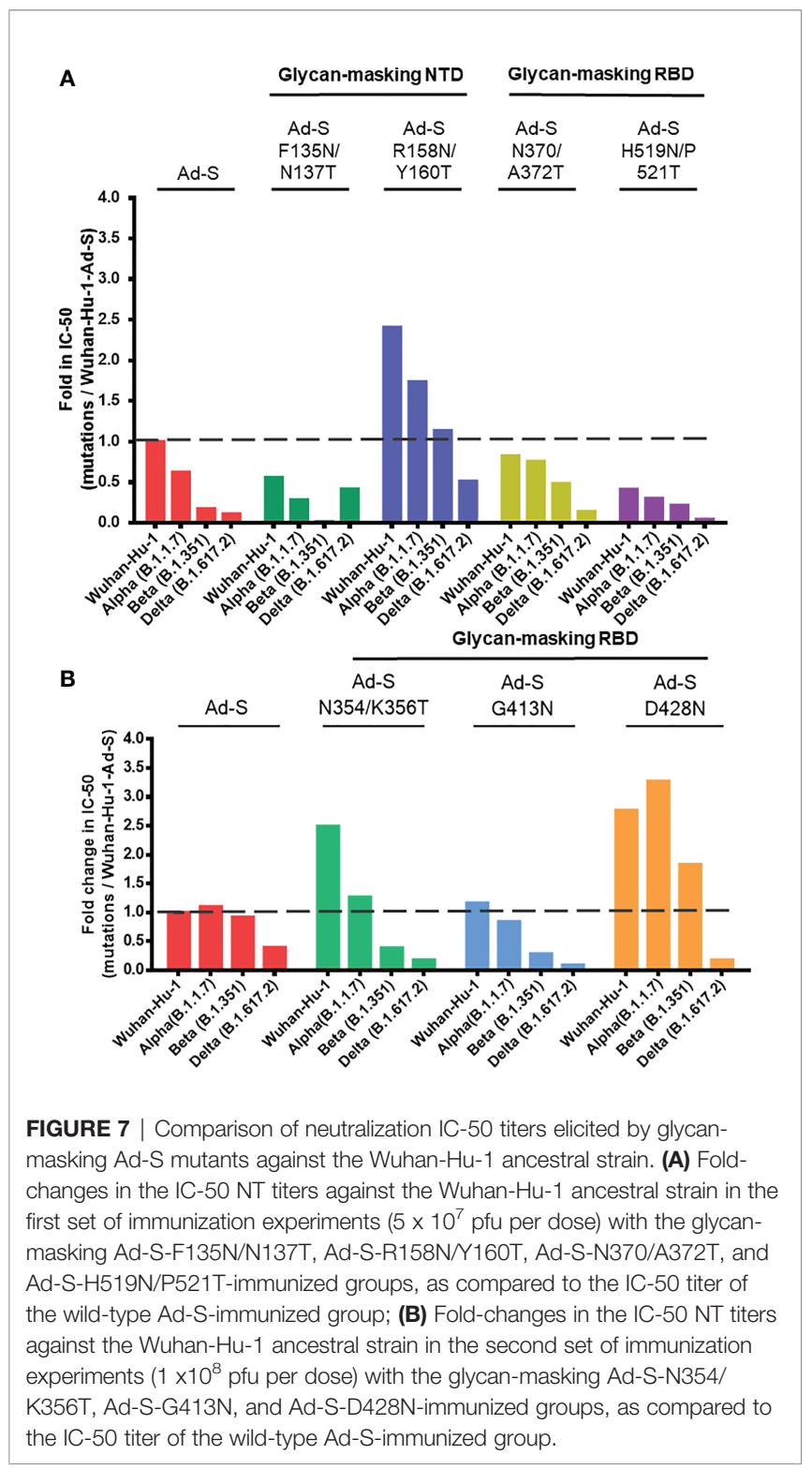

NTD were found to increase the neutralization titers against the Delta (B.1.617.2) variant (Figure 6D). Therefore, only the glycanmasking Ad-S-R158N/Y160T in NTD elicited broadly neutralizing antibody titers against Alpha (B.1.1.7), Beta (B.1.351), and Delta (B.1.617.2) variants. It is possible that the refocused antibodies using the glycan-masking Ad-S-R158N/Y160T antigen may target the NTD neutralizing epitopes in the N3 and N5 loops as recently reported $(34,35)$. One NTD neutralizing epitope has been shown to associate with the hydrophilic interactions of K147 and K150, the hydrophobic and/or $\pi-\pi$ interactions of Y145 and W152, and the Nlinked glycosylation site of N149 on the N3 loop of the NTD (34). It is also possible that the glycan-masking R158N/Y160T in the NTD interacts spatially with the RBD of another S1 monomer to affect the RBD up and down conformational structures $(36,37)$. The C-type lectins such as L-SIGN and DC-SIGN have been shown to function as attachment receptors by enhancing ACE2-mediated infection, and monoclonal antibodies to NTD or the RBD conserved site can effectively block lectin-facilitated infection (38). Additionally, the unique $\mathrm{N}$-glycan on N149 of NTD can directly bind to the L-SIGN/ DC-SIGN lectins as a non-ACE2 receptor for SARS-CoV-2 virus infection (39). Another report was shown for the N92 glycan on NTD that can enhance the binding to the L-SIGN lectin to interact with the ACE2 receptor to further facilitate SARS-CoV-2 virus entry (40). It is possible that the glycan-masking Ad-S-R158N/ Y160T site which is nearby the N3 loop on NTD (Supplementary Figure 1) can enhance targeting these epitopes to elicit neutralizing antibodies to block the L-SIGN/DC-SIGN receptor binding and/or the interaction between the L-SIGN/DC-SIGN lectin co-receptor with the ACE2 receptor binding for SARS-CoV-2 infection.

Our present findings demonstrated that the glycan-masking Ad-S-R158N/Y160T in NTD resulted in a 2.8-fold, 6.5-fold, and 4.6-fold increase, respectively, in the IC-50 titers against the Alpha (B.1.1.7), Beta (B.1.351) and Delta (B.1.617.2) variants, respectively. The glycan-masking Ad-S-D428N in RBD resulted in a 3.0-fold and 2.0-fold increase, respectively, in the IC-50 NT titer against the Alpha (B.1.1.7) and Beta (B.1.351) variants. The glycan-masking Ad-S-R158N/Y160T site is close to the del 156157 of the Delta (B.1.617.2) variant and the del 143 and $144 \mathrm{~V}$ mutation of the Alpha (B.1.1.7) in the N3 loop on NTD (Supplementary Figure 1). The glycan-masking Ad-S-D428N was nearby the K417N mutation of the Beta (B.1.351) variant in the C7 loop on RBD (Supplementary Figure 1). For the Alpha (B.1.1.7) and Beta (B.1.351) variants, the del 69-70, del 144, and del 242-244 deletions in NTD and the K417N/T, E484K, and N501Y mutations in RBD have been shown to increase ACE2 binding affinity and evade antibody-mediated immunity (7-15). It is likely that selective pressures on the NTD and RBD epitopes of the S1 subunit may ultimately result in immune-evasion variants. Our present findings demonstrated the use of glycanmasking mutations in the neutralization-sensitive NTD and RBD epitopes of S1 subunit can refocus antibody responses to the broadly neutralizing epitope domains to overcome the immuneevasion variants. Therefore, glycan-masking the site-specific NTD and RBD epitopes may help develop universal COVID19 vaccines against current and future emerging SARS-CoV2 variants.

\section{DATA AVAILABILITY STATEMENT}

The raw data supporting the conclusions of this article will be made available by the authors, without undue reservation.

\section{ETHICS STATEMENT}

All experiments were conducted in accordance with the guidelines of the Laboratory Animal Center of the National Tsing Hua University (NTHU). Animal use protocols were reviewed and approved by the NTHU Institutional Animal Care and Use Committee (approval no. 109047). 


\section{AUTHOR CONTRIBUTIONS}

Conceptualization: W-SL, I-CC, H-CC, and S-CW. Formal analysis: W-SL, I-CC, and H-CC. Funding acquisition: Y-CL and S-CW. Investigation: W-SL, I-CC, and H-CC. Supervision: S-CW. Writing: S-CW. All authors provided feedback to the final draft. All authors contributed to the article and approved the submitted version.

\section{FUNDING}

This work was supported by the Ministry of Science and Technology, Taiwan (MOST 109-2926-B-030-001, MOST109-2313-B-007-001MY2, MOST109-2327-B-007-003) and National Tsing Hua University, Taiwan (109R2807E1, 110Q2805E1).

\section{REFERENCES}

1. Lythgoe KA, Hall M, Ferretti L, de Cesare M, MacIntyre-Cockett G, Trebes A, et al. SARS-CoV-2 Within-Host Diversity and Transmission. Science (2021) 372(6539):eabg0821. doi: 10.1126/science.abg0821

2. ViralZone SARS-CoV-2 Circulating Variants. Available at: https://viralzone. expasy.org/9556.

3. Altmann DM, Boyton RJ, Beale R. Immunity to SARS-CoV-2 Variants of Concern. Science (2021) 371(6534):1102-4. doi: 10.1126/science.abg7404

4. Hastie KM, Li H, Bedinger D, Schendel SL, Dennison SM, Li K, et al. Defining Variant-Resistant Epitopes Targeted by SARS-CoV-2 Antibodies: A Global Consortium Study. Science (2021) 374(6566):472-8. doi: 10.1126/science.abh2315

5. McCallum M, De Marco A, Lempp FA, Tortorici MA, Pinto D, Walls AC, et al. NTerminal Domain Antigenic Mapping Reveals a Site of Vulnerability for SARSCoV-2. Cell (2021) 184(9):2332-47.e16. doi: 10.1016/j.cell.2021.03.028

6. Mlcochova P, Kemp S, Dhar MS, Papa G, Meng B, Ferreira IATM, et al. SARS-CoV-2 B.1.617.2 Delta Variant Replication and Immune Evasion. Nature (2021) 599(7883):114-9. doi: 10.1038/s41586-021-03944-y

7. Thomson EC, Rosen LE, Shepherd JG, Spreafico R, da Silva Filipe A, Wojcechowskyj JA, et al. Circulating SARS-CoV-2 Spike N439K Variants Maintain Fitness While Evading Antibody-Mediated Immunity. Cell (2021) 184(5):1171-1187.e20. doi: 10.1016/j.cell.2021.01.037

8. Yuan M, Huang D, Lee C-CD, Wu NC, Jackson AM, Zhu X, et al. Structural and Functional Ramifications of Antigenic Drift in Recent SARS-CoV-2 Variants. Science (2021) 373(6556):818-23. doi: 10.1126/science.abh1139

9. Wang P, Nair MS, Liu L, Iketani S, Luo Y, Guo Y, et al. Antibody Resistance of SARS-CoV-2 Variants B.1.351 and B.1.1.7. Nature (2021) 593(7857):130-5. doi: 10.1038/s41586-021-03398-2

10. Wang Z, Schmidt F, Weisblum Y, Muecksch F, Barnes CO, Finkin S, et al. mRNA Vaccine-Elicited Antibodies to SARS-CoV-2 and Circulating Variants. Nature (2021) 592(7855):616-22. doi: 10.1038/s41586-021-03324-6

11. Chen RE, Zhang X, Case JB, Winkler ES, Liu Y, VanBlargan LA, et al. Resistance of SARS-CoV-2 Variants to Neutralization by Monoclonal and Serum-Derived Polyclonal Antibodies. Nat Med (2021) 27(4):717-26. doi: 10.1038/s41591-021-01294-w

12. Collier DA, De Marco A, Ferreira IATM, Meng B, Datir RP, Walls AC, et al. Sensitivity of SARS-CoV-2 B.1.1.7 to mRNA Vaccine-Elicited Antibodies. Nature (2021) 593(7857):136-41. doi: 10.1038/s41586-021-03412-7

13. Liu Y, Liu J, Xia H, Zhang X, Fontes-Garfias CR, Swanson KA, et al. Neutralizing Activity of BNT162b2-Elicited Serum. N Engl J Med (2021) 384(15):1466-8. doi: 10.1056/NEJMc2102017

14. Wibmer CK, Ayres F, Hermanus T, Madzivhandila M, Kgagudi P, Oosthuysen B, et al. SARS-CoV-2 501y.V2 Escapes Neutralization by South African COVID-19 Donor Plasma. Nat Med (2021) 27(4):622-5. doi: 10.1038/s41591-021-01285-X

\section{ACKNOWLEDGMENTS}

We thank the RNAi core facility at Academia Sinica for performing the SARS-CoV-2 S-pseudotyped neutralization assay.

\section{SUPPLEMENTARY MATERIAL}

The Supplementary Material for this article can be found online at: https://www.frontiersin.org/articles/10.3389/fimmu.2021. 795741/full\#supplementary-material

Supplementary Figure 1 | Amino acid sequence alignment of SARS-CoV-2 Wuhan-Hu-1, Alpha (B.1.1.7), Beta (B.1.351), and Delta (B.1.617.2) strains. The NTD loops (blue), the CTD loops (green), and the receptor-binding motif (RBM, red) in the S protein are indicated. The glycan-masking sites (yellow) and variant mutation sites (pink) are marked. The CTD loops are the C-terminal domain loops of S1 subunit.

15. Madhi SA, Baillie V, Cutland CL, Voysey M, Koen AL, Fairlie L, et al. Efficacy of the ChAdOx1 Ncov-19 Covid-19 Vaccine Against the B.1.351 Variant. N Engl J Med (2021) 384(20):1885-98. doi: 10.1056/NEJMoa2102214

16. Nara PL, Tobin GJ, Chaudhuri AR, Trujillo JD, Lin G, Cho MW, et al. How can Vaccines Against Influenza and Other Viral Diseases be Made More Effective? PloS Biol (2010) 8:e1000571. doi: 10.1371/journal.pbio.1000571

17. Zolla-Pazner S, Cardozo T. Structure-Function Relationships of HIV-1 Envelope Sequence-Variable Regions Refocus Vaccine Design. Nat Rev Immunol (2010) 10:527-35. doi: 10.1038/nri2801

18. Delves PJ, Lund T, Roitt IM. Can Epitope-Focused Vaccines Select Advantageous Immune Responses? Mol Med Today (1997) 3(2):55-60. doi: 10.1016/S1357-4310(96)20036-X

19. Garrity RR, Rimmelzwaan G, Minassian A, Tsai WP, Lin G, de Jong JJ, et al. Refocusing Neutralizing Antibody Response by Targeted Dampening of an Immunodominant Epitope. J Immunol (1997) 159(1):279-89.

20. Chiesa MD, Martensen PM, Simmons C, Porakishvili N, Justesen J, Dougan G, et al. Refocusing of B-Cell Respones Following a Single Amino Acid Substitution in an Antigen. Immunology (2001) 103(2):172-8. doi: 10.1046/ j.1365-2567.2001.01242.x

21. Pantophlet R, Wilson IA, Burton DR. Hyperglycosylated Mutants of Human Immunodeficiency Virus (HIV) Type 1 Monomeric Gp120 as Novel Antigens for HIV Vaccine Design. J Virol (2003) 77(10):5889-901. doi: 10.1128/ JVI.77.10.5889-5901.2003

22. Selvarajah S, Puffer B, Pantophlet R, Law M, Doms RW, Burton DR. Comparing Antigenicity and Immunogenicity of Engineered Gp120. J Virol (2005) 79(19):12148-63. doi: 10.1128/JVI.79.19.12148-12163.2005

23. Duan H, Chen X, Boyington JC, Cheng C, Zhang Y, Jafari AJ, et al. Glycan Masking Focuses Immune Responses to the HIV-1 CD4-Binding Site and Enhances Elicitation of VRC01-Class Precursor Antibodies. Immunity (2018) 49(2):301-11.e5. doi: 10.1016/j.immuni.2018.07.005

24. Tobin GJ, Trujillo JD, Bushnell RV, Lin G, Chaudhuri AR, Long J, et al. Deceptive Imprinting and Immune Refocusing in Vaccine Design. Vaccine (2008) 26(49):6189-99. doi: 10.1016/j.vaccine.2008.09.080

25. Lin SC, Lin YF, Chong P, Wu SC. Broader Neutralizing Antibodies Against H5N1 Viruses Using Prime-Boost Immunization of Hyperglycosylated Hemagglutinin DNA and Virus-Like Particles. PloS One (2012) 7(6):e39075. doi: 10.1371/journal.pone.0039075

26. Lin SC, Liu WC, Jan JT, Wu SC. Glycan Masking of Hemagglutinin for Adenovirus Vector and Recombinant Protein Immunizations Elicits Broadly Neutralizing Antibodies Against H5N1 Avian Influenza Viruses. PloS One (2014) 9(3):e92822. doi: 10.1371/journal.pone.0092822

27. Chen TH, Liu WC, Lin CY, Liu CC, Jan JT, Spearman M, et al. GlycanMasking Hemagglutinin Antigens From Stable CHO Cell Clones for H5N1 Avian Influenza Vaccine Development. Biotechnol Bioeng (2019) 116(3):598609. doi: $10.1002 /$ bit. 26810 
28. Lin HH, Yang SP, Tsai MJ, Lin GC, Wu HC, Wu SC. Dengue and Zika Virus Domain III-Flagellin Fusion and Glycan-Masking E Antigen for Prime-Boost Immunization. Theranostics (2019) 9(16):4811-26. doi: 10.7150/thno.35919

29. Du L, Tai W, Yang Y, Zhao G, Zhu Q, Sun S, et al. Introduction of Neutralizing Immunogenicity Index to the Rational Design of MERS Coronavirus Subunit Vaccines. Nat Commun (2016) 7:13473. doi: 10.1038/ncomms13473

30. Walls AC, Park YJ, Tortorici MA, Wall A, McGuire AT, Veesler D. Structure, Function, and Antigenicity of the SARS-CoV-2 Spike Glycoprotein. Cell (2020) 181(2):281-92.e6. doi: 10.1016/j.cell.2020.02.058

31. Wrapp D, Wang N, Corbett KS, Goldsmith JA, Hsieh CL, Abiona O, et al. Cryo-EM Structure of the 2019-Ncov Spike in the Prefusion Conformation. Science (2020) 367(6483):1260-3. doi: 10.1126/science.abb2507

32. Yao H, Song Y, Chen Y, Wu N, Xu J, Sun C, et al. Molecular Architecture of the SARS-CoV-2 Virus. Cell (2020) 183(3):730-8.e13. doi: 10.1016/ j.cell.2020.09.018

33. Watanabe Y, Allen JD, Wrapp D, McLellan JS, Crispin M. Site-Specific Glycan Analysis of the SARS-CoV-2 Spike. Science (2020) 369(6501):330-3. doi: 10.1126/science.abb9983

34. Chi X, Yan R, Zhang J, Zhang G, Zhang Y, Hao M, et al. A Neutralizing Human Antibody Binds to the N-Terminal Domain of the Spike Protein of SARS-CoV-2. Science (2020) 369(6504):650-5. doi: 10.1126/science.abc6952

35. Suryadevara N, Shrihari S, Gilchuk P, VanBlargan LA, Binshtein E, Zost SJ, et al. Neutralizing and Protective Human Monoclonal Antibodies Recognizing the N-Terminal Domain of the SARS-CoV-2 Spike Protein. Cell (2021) 184(9):2316-31.e15. doi: 10.1016/j.cell.2021.03.029

36. Cai Y, Zhang J, Xiao T, Peng H, Sterling SM, Walsh RM Jr, et al. Distinct Conformational States of SARS-CoV-2 Spike Protein. Science (2020) 369 (6511):1586-92. doi: 10.1126/science.abd4251

37. Ray D, Le L, Andricioaei I. Distant Residues Modulate Conformational Opening in SARS-CoV-2 Spike Protein. Proc Natl Acad Sci U.S.A. (2021) 118(43):e2100943118. doi: 10.1073/pnas.2100943118
38. Lempp FA, Soriaga LB, Montiel-Ruiz M, Benigni F, Noack J, Park YJ, et al. Lectins Enhance SARS-CoV-2 Infection and Influence Neutralizing Antibodies. Nature (2021) 598(7880):342-7. doi: 10.1038/s41586-02103925-1

39. Soh WT, Liu Y, Nakayama EE, Ono C, Torii S, Nakagami H, et al. The N-Terminal Domain of Spike Glycoprotein Mediates SARS-CoV-2 Infection by Associating With L-SIGN and DC-SIGN. bioRxiv (2020). doi: 10.1101/ 2020.11.05.369264

40. Amraei R, Yin W, Napoleon MA, Suder EL, Berrigan J, Zhao Q, et al. CD209L/ L-SIGN and CD209/DC-SIGN Act as Receptors for SARS-CoV-2. ACS Cent Sci (2021) 7(7):1156-65. doi: 10.1021/acscentsci.0c01537

Conflict of Interest: S-CW, W-SL, I-CC and Y-CL are co-inventors on a U.S. provisional patent (No. 63/169,268) filed on April 1, 2021.

The remaining author declares that the research was conducted in the absence of any commercial or financial relationships that could be construed as a potential conflict of interest.

Publisher's Note: All claims expressed in this article are solely those of the authors and do not necessarily represent those of their affiliated organizations, or those of the publisher, the editors and the reviewers. Any product that may be evaluated in this article, or claim that may be made by its manufacturer, is not guaranteed or endorsed by the publisher.

Copyright (c) 2021 Lin, Chen, Chen, Lee and Wu. This is an open-access article distributed under the terms of the Creative Commons Attribution License (CC BY). The use, distribution or reproduction in other forums is permitted, provided the original author(s) and the copyright owner(s) are credited and that the original publication in this journal is cited, in accordance with accepted academic practice. No use, distribution or reproduction is permitted which does not comply with these terms. 